\title{
Interaction of cyclodextrins with pyrene-modified polyacrylamide in a mixed solvent of water and dimethyl sulfoxide as studied by steady-state fluorescence
}

\author{
Akihito Hashidzume $^{1}$, Yongtai Zheng ${ }^{1}$ and Akira Harada*1,2
}

\section{Full Research Paper}

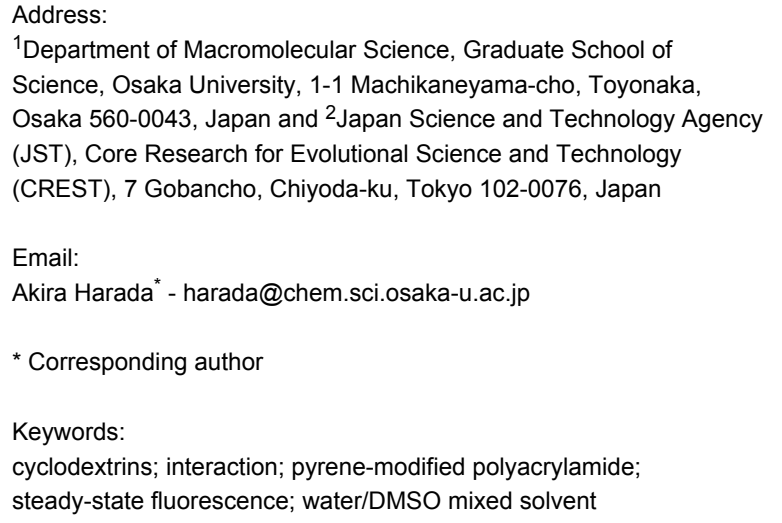

${ }^{1}$ Department of Macromolecular Science, Graduate School of Science, Osaka University, 1-1 Machikaneyama-cho, Toyonaka, Osaka 560-0043, Japan and ${ }^{2}$ Japan Science and Technology Agency (JST), Core Research for Evolutional Science and Technology (CREST), 7 Gobancho, Chiyoda-ku, Tokyo 102-0076, Japan

Email:

Akira Harada* - harada@chem.sci.osaka-u.ac.jp

* Corresponding author

Keywords:

cyclodextrins; interaction; pyrene-modified polyacrylamide; steady-state fluorescence; water/DMSO mixed solvent

\author{
Beilstein J. Org. Chem. 2012, 8, 1312-1317. \\ doi:10.3762/bjoc. 8.150 \\ Received: 20 April 2012 \\ Accepted: 31 July 2012 \\ Published: 16 August 2012 \\ This article is part of the Thematic Series "Superstructures with \\ cyclodextrins: Chemistry and applications". \\ Guest Editor: H. Ritter \\ (c) 2012 Hashidzume et al; licensee Beilstein-Institut. \\ License and terms: see end of document.
}

\begin{abstract}
The interaction of $\beta$ - and $\gamma$-cyclodextrins ( $\beta-\mathrm{CD}$ and $\gamma-\mathrm{CD}$, respectively) with polyacrylamide modified with pyrenyl (Py) residues (pAAmPy) was investigated in a mixed solvent of water and dimethyl sulfoxide (DMSO) by steady-state fluorescence. In the absence of $\mathrm{CD}$, the fluorescence spectra indicated that the formation of Py dimers became less favorable with increasing volume fraction of DMSO $\left(x_{\mathrm{DMSO}}\right)$. The fluorescence spectra at varying $x_{\mathrm{DMSO}}$ and CD concentrations indicated that $\beta-\mathrm{CD}$ and $\gamma-\mathrm{CD}$ included monomeric and dimeric Py residues, respectively. Using the fluorescence spectra, equilibrium constants of the formation of Py dimers and the complexation of $\beta-\mathrm{CD}$ and $\gamma-\mathrm{CD}$ with Py residues were roughly estimated based on simplified equilibrium schemes.
\end{abstract}

\section{Introduction}

Cyclodextrins (CDs) are cyclic oligomers composed of glucopyranose units linked through $\alpha$-1,4-glycoside bonding. They bear a tapered structure with a narrower rim of primary hydroxy groups and a wider rim of secondary hydroxy groups. CDs of 6,7 , and 8 glucopyranose units are called $\alpha-C D, \beta-C D$, and $\gamma-\mathrm{CD}$, respectively. CDs have a hydrophilic exterior and a rather hydrophobic cavity, and thus, recognize guest compounds of a size and shape matching their cavity, to form inclusion complexes [1-5]. Since CDs are nontoxic, they have been utilized in a variety of fields, including food additives, cosmetics, and personal care items [6-12]. In the past decade, the formation of inclusion complexes of CDs with guest residues attached on water-soluble polymers has attracted increasing interest from a number of research groups because these systems are applicable to stimuli-responsive systems [13-18]. 
We have been working on the interaction of CDs with watersoluble polymers bearing various guest residues, including linear, branched, and cyclic aliphatics, as well as aromatics [1921], and realized stimuli-responsive hydrogels [22-27] and macroscopic assemblies based on molecular recognition [2831]. Aromatic residues absorb light to become excited, and subsequently they can transfer energy and electrons. The interaction of CDs with water-soluble polymers carrying aromatic residues may allow one to construct functional systems that convert photo energy based on molecular recognition. Among aromatic compounds, pyrene is the most examined as a fluorescence probe or label because it shows a relatively high fluorescence quantum yield and a relatively long fluorescence lifetime in both monomer and excimer states $[32,33]$. Since pyrene is very hydrophobic, it may tend to form aggregates, e.g., dimers, in aqueous solutions. It is also known that pyrene forms inclusion complexes with $\beta-\mathrm{CD}$ and $\gamma-\mathrm{CD}$ in different manners; $\beta$-CD includes monomeric pyrene whereas $\gamma$-CD includes dimeric pyrene [34-36]. Recently, we have demonstrated this selectivity switching on macroscopic molecular recognition for polyacrylamide-based gels carrying pyrenyl (Py) and CD residues, by changing the composition of a mixed solvent of water and dimethyl sulfoxide (DMSO) [37]. In the present study, the interaction of $\beta-\mathrm{CD}$ and $\gamma$-CD with Py-modified polyacrylamide (pAAmPy, Scheme 1) was investigated in the water/ DMSO mixed solvent of varying composition by steady-state fluorescence to elucidate the mechanism of the selectivity switching.

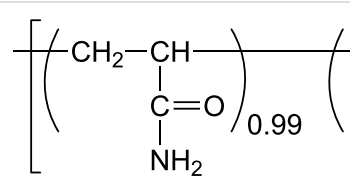

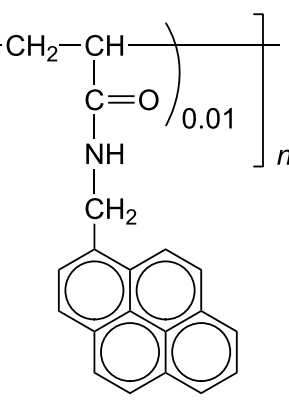

Scheme 1: Structure of pAAmPy.

\section{Results}

Figure 1a demonstrates the steady-state fluorescence spectra measured for $0.04 \mathrm{~g} \mathrm{~L}^{-1} \mathrm{pAAmPy}(5 \mu \mathrm{M}$ in Py residue) at varying volume fractions of DMSO ( $\left.x_{\mathrm{DMSO}}\right)$ in the water/ DMSO mixed solvent in the absence of CD. At $x_{\mathrm{DMSO}}=0$ (i.e., in water), the spectrum exhibits not only emission bands ascribable to monomeric Py in the region of $370-430 \mathrm{~nm}$, but also a broad band assignable to a Py excimer around $480 \mathrm{~nm}$, indicating that Py residues tend to form dimers because of the hydrophobicity. It is likely that Py residues associate intramole- cularly under the dilute conditions $\left(0.04 \mathrm{~g} \mathrm{~L}^{-1}\right)$ in this study. These spectra indicate that the intensity of excimer fluorescence decreases whereas that of monomer fluorescence increases with increasing $x_{\text {DMSO }}$. This observation indicates that the formation of Py dimer becomes less favorable, because the Py residue becomes more solvophilic with $x_{\text {DMSO }}$. Using the spectra, the ratios $\left(I_{480} / I_{376}\right)$ of the intensities at 480 and $376 \mathrm{~nm}$, which are predominantly due to the Py excimer and monomer, respectively, were calculated and plotted in Figure $1 \mathrm{~b}$ against $x_{\text {DMSO }} I_{480} / I_{376}$ decreases monotonously from 0.125 to 0.025 with increasing $x_{\text {DMSO }}$ from 0 to 1 .
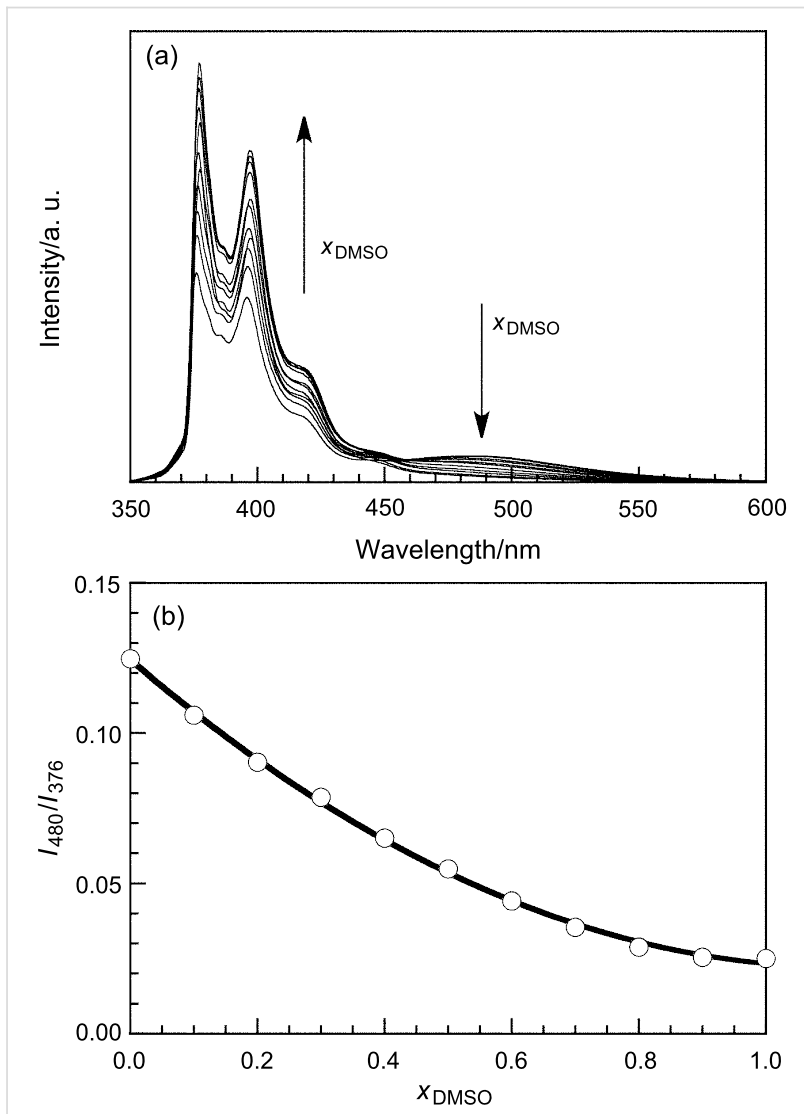

Figure 1: Steady-state fluorescence spectra for $0.04 \mathrm{~g} \mathrm{~L}^{-1} \mathrm{pAAmPy}$ at varying $x_{\mathrm{DMSO}}$ from 0 to 1 with excitation at $335 \mathrm{~nm}$ (a) and $I_{480} / I_{376}$ as a function of $x_{\mathrm{DMSO}}(\mathrm{b})$.

The interaction of $\beta-C D$ and $\gamma-C D$ with pAAmPy was also investigated at varying $x_{\text {DMSO }}$ by steady-state fluorescence. Figure 2 exhibits fluorescence spectra for the $\beta-\mathrm{CD} / \mathrm{pAAmPy}$ system at $x_{\mathrm{DMSO}}=0.1$ and for the $\gamma-\mathrm{CD} / \mathrm{pAAmPy}$ system at $x_{\text {DMSO }}=0$ as typical examples, showing remarkable tendencies. In the spectra of the $\beta-\mathrm{CD} / \mathrm{pAAmPy}$ system at $x_{\mathrm{DMSO}}=0.1$, the intensity of Py excimer fluorescence decreases whereas that of Py monomer fluorescence increases with the increasing concentration of $\mathrm{CD}\left([\mathrm{CD}]_{0}\right)$, indicating that $\beta$-CD forms inclusion complexes with monomeric Py residues, and dimeric Py 

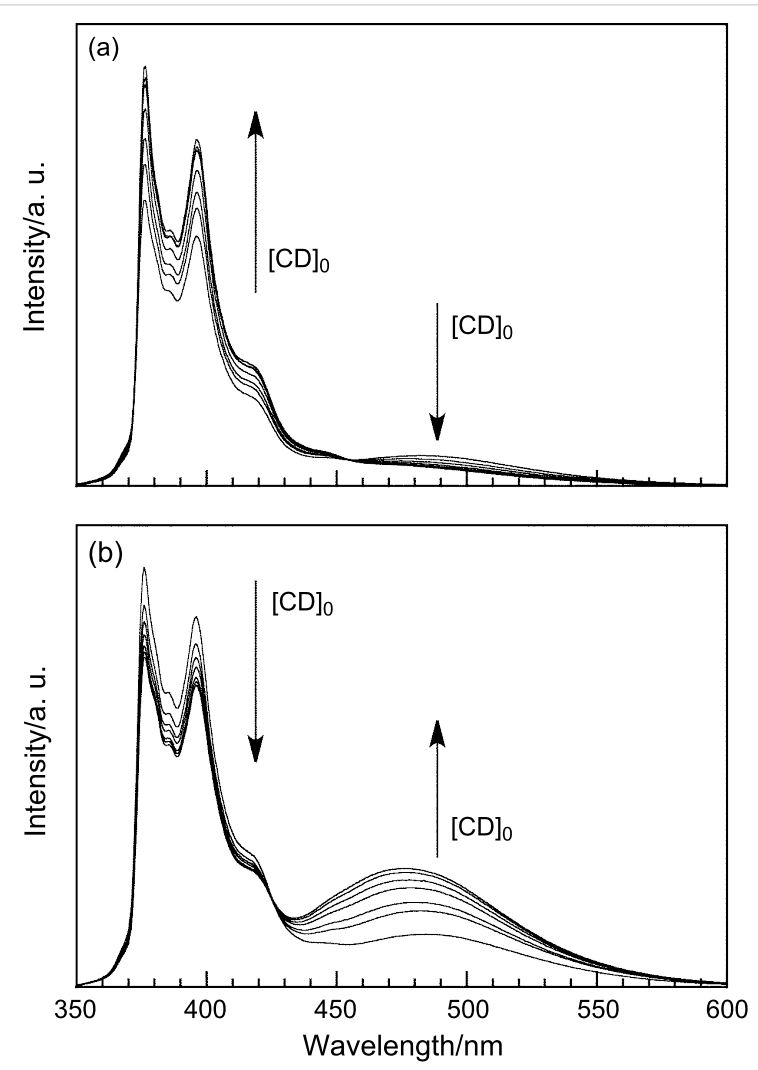

Figure 2: Steady-state fluorescence spectra for $0.04 \mathrm{~g} \mathrm{~L}^{-1} \mathrm{pAAmPy}$ with excitation at $335 \mathrm{~nm}$ in the presence of varying concentrations of $\beta-C D$ at $x_{\text {DMSO }}=0.1(a)$ and of $y-C D$ at $x_{\text {DMSO }}=0(b)$.

residues dissociate to the monomers. In the spectra of the $\gamma$-CD/pAAmPy system at $x_{\mathrm{DMSO}}=0$, on the other hand, the intensity of the excimer fluorescence increases whereas that of the monomer fluorescence decreases with increasing $[\mathrm{CD}]_{0}$, indicating that $\gamma$-CD forms inclusion complexes with dimeric Py residues, and monomeric Py residues further associate to form the dimers. Using the steady-state fluorescence spectra, $I_{480} / I_{376}$ values were calculated. Figure 3 compares $I_{480} / I_{376}$ as a function of $[\mathrm{CD}]_{0}$ for the $\beta-\mathrm{CD} / \mathrm{pAAmPy}$ system at $x_{\mathrm{DMSO}}=$ $0.1-0.6$ and for the $\gamma-\mathrm{CD} / \mathrm{pAAmPy}$ system at $x_{\mathrm{DMSO}}=0-0.2$. At other $x_{\text {DMSO }}, I_{480} / I_{376}$ was practically independent of $[C D]_{0}$, indicative of no significant interaction of $\beta-C D$ or $\gamma-C D$ with pAAmPy. For the $\beta$-CD/pAAmPy system (Figure $3 a$ ), $I_{480} / I_{376}$ decreases with increasing $[\mathrm{CD}]_{0}$ at $x_{\mathrm{DMSO}}=0.1-0.6$. For the $\gamma$-CD/pAAmPy system (Figure $3 b$ ), on the other hand, $I_{480} / I_{376}$ increases with $[\mathrm{CD}]_{0}$ at $x_{\mathrm{DMSO}}=0-0.2$.

\section{Discussion}

Detailed study of the equilibria of the inclusion complex formation of CDs with Py-modified water-soluble polymers, including the formation of the dynamic excimer, requires not only steady-state fluorescence measurements but also timeresolved fluorescence measurements [38-43]. In this study,
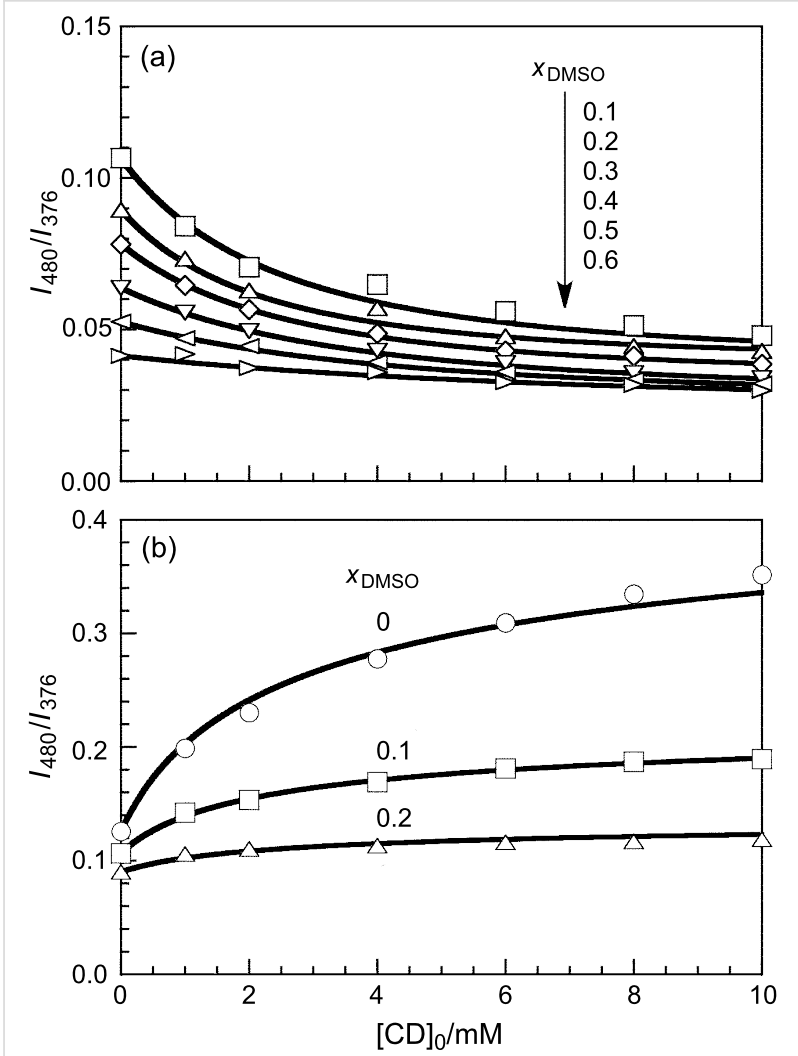

Figure 3: $I_{480} / /_{376}$ as a function of $[C D]_{0}$ for $\beta-C D / p A A m P y(a)$ and $\mathrm{Y}-\mathrm{CD} / \mathrm{pAAmPy}(\mathrm{b})$ at different $x_{\mathrm{DMSO}}$.

however, equilibrium constants are roughly estimated by analyzing the steady-state fluorescence data, assuming that dynamic excimer formation is negligible. In the absence of $\mathrm{CD}$, $I_{480} / I_{376}$ for pAAmPy decreases from 0.125 to 0.025 with increasing $x_{\mathrm{DMSO}}$ from 0 to 1 . It should be noted here that the fluorescence of the Py monomer is dominant compared to that of the Py excimer even at $x_{\mathrm{DMSO}}=0$ (i.e., in water), implying that there are a significant fraction of Py residues $\left(\mathrm{Py}^{\circ}\right)$ that cannot form Py dimers $\left(\mathrm{Py}_{2}\right)$. Since the steady-state fluorescence measurements were performed under dilute conditions in this study, most of the $\mathrm{Py}_{2}$ were formed intramolecularly. Thus, Py residues in pAAmPy carrying a Py residue may not form $\mathrm{Py}_{2}$. The fraction of $\mathrm{Py}^{\circ}$ is defined as $f$. Scheme 2a indicates a simplified equilibrium of the formation of $\mathrm{Py}_{2}$ from two $\mathrm{Py}$ residues. On the basis of the derivation of equations in the Supporting Information File 1, the equilibrium constant for the $\mathrm{Py}_{2}$ formation $\left(K_{\mathrm{Py}}\right)$ can be calculated as

$$
K_{\mathrm{Py}}=\frac{x^{2}-1}{8(1-f)[\mathrm{Py}]_{0}}
$$

where $[\mathrm{Py}]_{0}$ is the total concentration of Py residue and $x$ as given in Equation 2. 


$$
x=\frac{2\left(\frac{I_{480}}{I_{376}} A_{1,376}-A_{1,480}\right)(f-2)[\mathrm{Py}]_{0}+\left(\frac{I_{480}}{I_{376}} A_{2.376}-A_{2.480}\right)(1-f)[\mathrm{Py}]_{0}-2\left(\frac{I_{480}}{I_{376}} B_{376}-B_{480}\right)}{2\left(\frac{I_{480}}{I_{376}} A_{1,376}-A_{1,480}\right) f[\mathrm{Py}]_{0}+\left(\frac{I_{480}}{I_{376}} A_{2.376}-A_{2,480}\right)(1-f)[\mathrm{Py}]_{0}-2\left(\frac{I_{480}}{I_{376}} B_{376}-B_{480}\right)}
$$

Here, $A_{1,376}, A_{1,480}, A_{2,376}$, and $A_{2,480}$ are constants corresponding to the products of the molar extinction coefficient and the fluorescence quantum yield (subscripts 1 and 2 indicate monomeric and dimeric Py residues, respectively, and subscripts 376 and 480 indicate the wavelengths), and $B_{376}$ and $B_{480}$ are constants corresponding to the background. If it is assumed that all the Py residues are in the monomer state at $x_{\mathrm{DMSO}}=1$ (i.e., in DMSO), $f=0.5, A_{2,480} / A_{1,376}=0.5$, and $B_{376}=B_{480}=0, K_{\mathrm{Py}}$ can be calculated as can be seen in Figure $4 \mathrm{a}$. This figure indicates that $K_{\mathrm{Py}}$ decreases monotonously from $6.2 \times 10^{4}$ to $0 \mathrm{M}^{-1}$ with increasing $x_{\mathrm{DMSO}}$ from 0 to 1 .

(a)

$$
\mathrm{Py}+\mathrm{Py} \stackrel{K_{\mathrm{Py}}}{\rightleftharpoons} \mathrm{Py}_{2}
$$

(b)

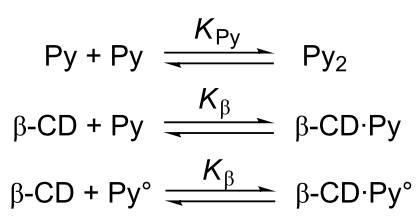

(c)

$$
\begin{aligned}
\mathrm{Py}+\mathrm{Py} & \stackrel{K_{\mathrm{Py}}}{\rightleftharpoons} \mathrm{Py}_{2} \\
\gamma-\mathrm{CD}+\mathrm{Py}_{2} \stackrel{K_{\gamma}}{\rightleftharpoons} & =\mathrm{CD} \cdot \mathrm{Py}_{2}
\end{aligned}
$$

Scheme 2: Simplified equilibria of CDs/pAAmPy systems.

In the $\beta-\mathrm{CD} / \mathrm{pAAmPy}$ system, $\beta-\mathrm{CD}$ forms inclusion complexes with both $\mathrm{Py}$ and $\mathrm{Py}^{\circ}$ (Scheme $2 \mathrm{~b}$ ). On the basis of the derivation described in the Supporting Information File 1, the concentrations of all species can be calculated by using the equilibrium constant $\left(K_{\beta}\right)$ for the inclusion complex formation, and $I_{480} / I_{376}$ can be also obtained as given in Equation 3 .

Here $[\mathrm{Py}],\left[\mathrm{Py}^{\circ}\right],[\mathrm{CD}],[\mathrm{CD} \cdot \mathrm{Py}]$, and $\left[\mathrm{CD} \cdot \mathrm{Py}^{\circ}\right]$ denote the concentrations of $\mathrm{Py}, \mathrm{Py}^{\circ}$, free $\mathrm{CD}$, and the complexes of $\mathrm{CD}$ with $\mathrm{Py}$ and with $\mathrm{Py}^{\circ}$, respectively, and $A_{1,376}^{\prime}$ and $A_{1,480}^{\prime}$ are constants. In this study, $A^{\prime}{ }_{1,480} / A^{\prime}{ }_{1,376}$ is fixed at 0.025

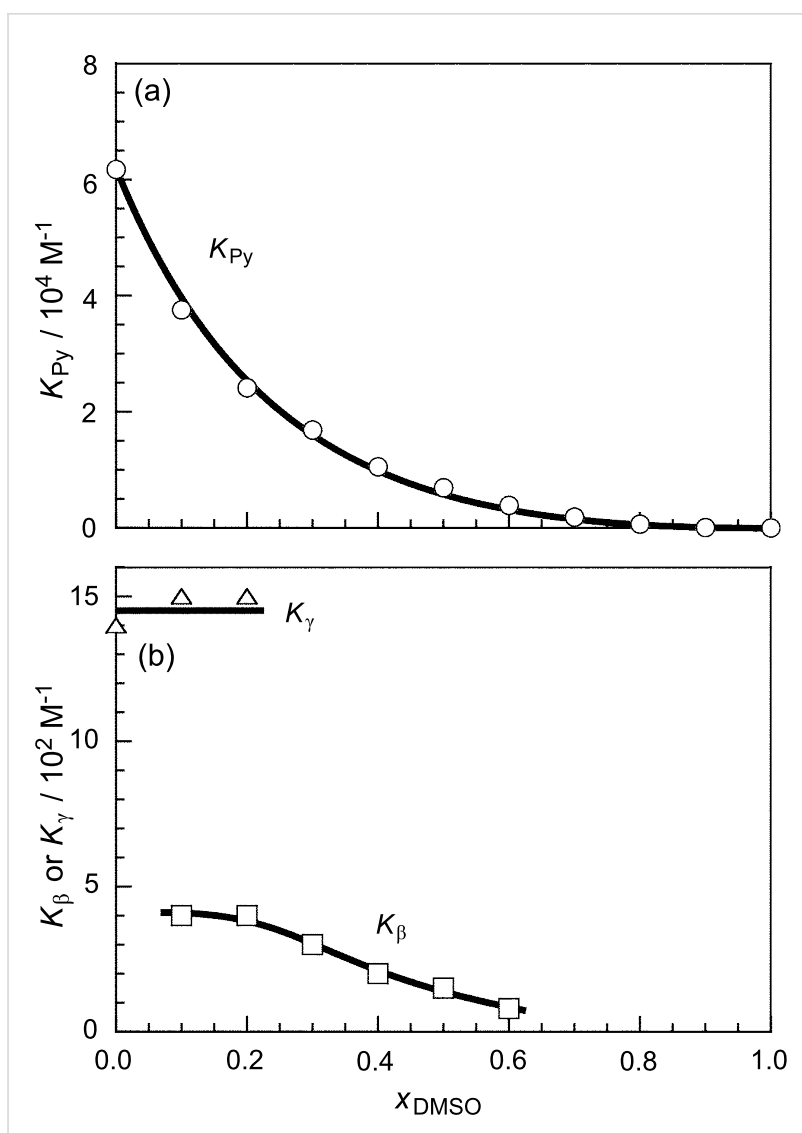

Figure 4: $K_{\mathrm{Py}}, K_{\beta}$, and $K_{\mathrm{y}}$ as a function of $x_{\mathrm{DMSO}}$.

(Supporting Information File 1). It is also likely that $A_{2,376}=0$. When $K_{\beta}$ and $A_{1,376}^{\prime} / A_{1,376}$ are chosen appropriately, the calculated $I_{480} / I_{376}$ values agree with the experimental data, as can be seen in Figure $3 \mathrm{a}$. The $K_{\beta}$ values were plotted in Figure $4 \mathrm{~b}$ against $x_{\text {DMSO }}$. As $x_{\text {DMSO }}$ increases from 0.1 to $0.6, K_{\beta}$ decreases from $4 \times 10^{2}$ to $8 \times 10^{1} \mathrm{M}^{-1}$. This observation indicates that the formation of inclusion complexes becomes less favorable with increasing $x_{\text {DMSO. }}$

In the $\gamma-\mathrm{CD} / \mathrm{pAAmPy}$ system, $\gamma-\mathrm{CD}$ forms inclusion complexes with $\mathrm{Py}_{2}$, in which $\mathrm{Py}^{\circ}$ is not involved. On the basis of the

$$
I_{480} / I_{376}=\frac{A_{1,480}\left([\mathrm{Py}]+\left[\mathrm{Py}^{\circ}\right]+A_{1,480}^{\prime}\left([\mathrm{CD} \cdot \mathrm{Py}]+\left[\mathrm{CD} \cdot \mathrm{Py}^{\circ}\right]\right)+A_{2,480}\left[\mathrm{Py}_{2}\right]+B_{480}\right.}{A_{1,376}\left([\mathrm{Py}]+\left[\mathrm{Py}^{\circ}\right]+A_{1,376}^{\prime}\left([\mathrm{CD} \cdot \mathrm{Py}]+\left[\mathrm{CD} \cdot \mathrm{Py}^{\circ}\right]\right)+A_{2,376}\left[\mathrm{Py}_{2}\right]+B_{376}\right.}
$$




$$
I_{480} / I_{376}=\frac{A_{1,480}\left([\mathrm{Py}]+\left[\mathrm{Py}^{\circ}\right]+A_{2,480}\left(\left[\mathrm{Py}_{2}\right]+A_{2,480}^{\prime}\left[\mathrm{CD} \cdot \mathrm{Py}_{2}\right]+B_{480}\right.\right.}{A_{1,376}\left([\mathrm{Py}]+\left[\mathrm{Py}^{\circ}\right]+A_{2,376}\left(\left[\mathrm{Py}_{2}\right]+A_{2,376}^{\prime}\left[\mathrm{CD} \cdot \mathrm{Py}_{2}\right]+B_{376}\right.\right.}
$$

derivation described in the Supporting Information File 1, the concentrations of all species can be calculated by using the equilibrium constant $\left(K_{\gamma}\right)$ for the inclusion complex formation, and $I_{480} / I_{376}$ can be also obtained as given in Equation 4.

Here $\left[\mathrm{CD} \cdot \mathrm{Py}_{2}\right]$ denotes the concentration of the complex of $\mathrm{CD}$ with $\mathrm{Py}_{2}$, and $A_{2,480}^{\prime}$ is a constant. It is also likely that $A_{2,376}=$ $A_{2,376}^{\prime}=0$. When $K_{\gamma}$ and $A_{2,480}^{\prime} / A_{1,376}$ are chosen appropriately, the $I_{480} / I_{376}$ values calculated agree with the experimental data, as can be seen in Figure $3 \mathrm{~b}$. The $K_{\gamma}$ values were also plotted in Figure $4 \mathrm{~b}$ against $x_{\mathrm{DMSO}}$. This figure indicates that $K_{\gamma}$ is practically constant (ca. $1.5 \times 10^{3} \mathrm{M}^{-1}$ ) independent of $x_{\mathrm{DMSO}}$ in the region of $0 \leq x_{\mathrm{DMSO}} \leq 0.2$.

It should be noted here that the values of $K_{\mathrm{Py}}, K_{\beta}$, and $K_{\gamma}$ were estimated rather qualitatively based on the simplified equilibria and a number of assumptions, but the $K_{\beta}$ and $K_{\gamma}$ values are in good agreement with the values reported for pyrene $\left(4.9 \times 10^{2}\right.$ and $1.1 \times 10^{3} \mathrm{M}^{-1}$ for $\beta-\mathrm{CD}$ and $\gamma-\mathrm{CD}$, respectively) [44].

\section{Experimental}

1-Pyrenemethylamine hydrochloride was purchased from Sigma-Aldrich Co. Ltd. Acryloyl chloride was obtained from Tokyo Chemical Industry Co. Ltd. Triethylamine, acrylamide (AAm), ammonium peroxodisulfate (APS), acetone, methanol, DMSO (spectroscopic grade), $\mathrm{NaHCO}_{3}$, and $\mathrm{NaOH}$ were purchased from Nacalai Tesque Inc. $N, N$-Dimethylformamide (DMF) and dichloromethane (DCM) were purified by utilizing a glass contour solvent dispensing system. Water was purified by a Millipore Milli-Q system. $\beta-\mathrm{CD}$ and $\gamma-\mathrm{CD}$ were purchased from Junsei Chemical Co. Ltd. and recrystallized twice from water before use. $N$-1-Pyrenylmethylacrylamide (APy) was prepared from 1-pyrenemethylamine hydrochloride and acryloyl chloride according to the procedure reported previously [37]. Other reagents were reagent grade and used without further purification.

The polymer (pAAmPy) was prepared by radical copolymerization of AAm and APy using APS as the initiator. A predetermined amount of AAm and APy were dissolved in DMF. After purging with dry argon for $30 \mathrm{~min}$, APS ( $3 \mathrm{mg}, 13 \mu \mathrm{mol})$ was added to the monomer solution. The reaction mixture was placed into a cuvette equipped with a stirrer and sealed. The cuvette was warmed with an oil bath thermostated at $60{ }^{\circ} \mathrm{C}$ overnight. The reaction mixture was poured into an excess of methanol to give a precipitate. The polymer obtained was recovered by filtration and dried under vacuum. The molecular weight of the polymer was estimated to be $4 \times 10^{3}$ by size exclusion chromatography (SEC), and the Py content was determined to be ca. $1 \mathrm{~mol} \%$ by ${ }^{1} \mathrm{H}$ NMR.

Steady-state fluorescence spectra were obtained on a HITACHI F-2500 spectrophotometer with excitation at $335 \mathrm{~nm}$ by using a $1 \mathrm{~cm}$ quartz cuvette. The slit widths for both excitation and emission sides were kept at $2.5 \mathrm{~nm}$ during measurement. SEC analysis was carried out at $40{ }^{\circ} \mathrm{C}$ on a TOSOH CCP \& 8020 system equipped with two TOSOH TSKgel $\alpha$-M columns connected in series, using formamide as the eluent at a flow rate of $0.3 \mathrm{~mL} \mathrm{~min}-1$. TOSOH UV-8020 and TOSOH RI-8021 detectors were used. The molecular weights were calibrated by polystyrene sulfonate sodium-salt samples (American Polymer Standards). ${ }^{1} \mathrm{H}$ NMR spectra were measured on a JEOL JNMECA500 spectrometer by using a mixed solvent of DMSO- $d_{6}$ and $\mathrm{D}_{2} \mathrm{O}(1 / 1, \mathrm{v} / \mathrm{v})$ as a solvent, and chemical shifts were referenced to the solvent value (i.e., $2.49 \mathrm{ppm}$ for DMSO).

\section{Supporting Information}

\section{Supporting Information File 1}

Equilibria for the CDs/pAAmPy systems.

[http://www.beilstein-journals.org/bjoc/content/ supplementary/1860-5397-8-150-S1.pdf]

\section{Acknowledgements}

The authors are grateful to Associate Professor Hiroyasu Yamaguchi and Assistant Professor Yoshinori Takashima, Department of Macromolecular Science, Graduate School of Science, Osaka University, for fruitful discussion and suggestions.

\section{References}

1. Bender, M. L.; Komiyama, M. Cyclodextrin Chemistry; Springer: Berlin, Germany, 1978.

2. Szejtli, J. Cyclodextrins and Their Inclusion Complexes; Akadémiai Kiadó: Budapest, Hungury, 1982.

3. Szejtli, J.; Osa, T., Eds. Cyclodextrins; Pergamon: Oxford, U.K., 1996.

4. Harada, A. In Large Ring Molecules; Semlyen, J. A., Ed.; Wiley \& Sons: Chichester, U.K., 1996; pp 407-432.

5. Dodziuk, H., Ed. Cyclodextrins and Their Complexes: Chemistry, Analytical Methods, Applications; Wiley-VCH: Weinheim, Germany, 2006. 
6. Uekama, K.; Hirayama, F.; Irie, T. Chem. Rev. 1998, 98, 2045-2076. doi:10.1021/cr970025p

7. Uekama, K.; Hirayama, F.; Arima, H. Pharmaceutical Applications of Cyclodextrins and Their Derivatives. In Cyclodextrins and Their Complexes: Chemistry, Analytical Methods, Applications; Dodziuk, H., Ed.; Wiley-VCH: Weinheim, Germany, 2006; pp 381-422. doi:10.1002/3527608982.ch14

8. Hashimoto, H. In Cyclodextrins and Their Complexes: Chemistry, Analytical Methods, Applications; Dodziuk, H., Ed.; Wiley-VCH: Weinheim, Germany, 2006; pp 452-459.

9. Szejtli, J.; Szente, L. Eur. J. Pharm. Biopharm. 2005, 61, 115-125. doi:10.1016/j.ejpb.2005.05.006

10. Brewster, M. E.; Loftsson, T. Adv. Drug Delivery Rev. 2007, 59, 645-666. doi:10.1016/j.addr.2007.05.012

11. Astray, G.; Gonzalez-Barreiro, C.; Mejuto, J. C.; Rial-Otero, R.; Simal-Gándara, J. Food Hydrocolloids 2009, 23, 1631-1640. doi:10.1016/j.foodhyd.2009.01.001

12. Loftsson, T.; Brewster, M. E. J. Pharm. Pharmacol. 2010, 62, 1607-1621. doi:10.1111/j.2042-7158.2010.01030.x

13. Wenz, G.; Han, B.-H.; Müller, A. Chem. Rev. 2006, 106, 782-817. doi:10.1021/cr970027+

14. Harada, A.; Hashidzume, A.; Yamaguchi, H.; Takashima, Y. Chem. Rev. 2009, 109, 5974-6023. doi:10.1021/cr9000622

15. Zhou, J.; Ritter, H. Polym. Chem. 2010, 1, 1552-1559. doi:10.1039/c0py00219d

16. Yuen, F.; Tam, K. C. Soft Matter 2010, 6, 4613-4630. doi:10.1039/c0sm00043d

17. Chen, G.; Jiang, M. Chem. Soc. Rev. 2011, 40, 2254-2266. doi:10.1039/c0cs00153h

18. Harada, A.; Hashidzume, A.; Yamaguchi, H.; Takashima, Y. Polymeric Materials; John Wiley \& Sons, Inc., 2012; pp 1-31.

19. Hashidzume, A.; Tomatsu, I.; Harada, A. Polymer 2006, 47, 6011-6027. doi:10.1016/j.polymer.2006.06.021

20. Harada, A.; Hashidzume, A. Aust. J. Chem. 2010, 63, 599-610. doi:10.1071/CH09609

21. Hashidzume, A.; Harada, A. Polym. Chem. 2011, 2, 2146-2154. doi:10.1039/c1py00162k

22. Tomatsu, I.; Hashidzume, A.; Harada, A. Macromolecules 2005, 38, 5223-5227. doi:10.1021/ma050670v

23. Tomatsu, I.; Hashidzume, A.; Harada, A. Macromol. Rapid Commun. 2006, 27, 238-241. doi:10.1002/marc. 200500793

24. Tomatsu, I.; Hashidzume, A.; Harada, A. J. Am. Chem. Soc. 2006, 128, 2226-2227. doi:10.1021/ja058345a

25. Tamesue, S.; Takashima, Y.; Yamaguchi, H.; Shinkai, S.; Harada, A. Angew. Chem., Int. Ed. 2010, 49, 7461-7464. doi:10.1002/anie.201003567

26. Tamesue, S.; Takashima, Y.; Yamaguchi, H.; Shinkai, S.; Harada, A. Eur. J. Org. Chem. 2011, 2801-2806. doi:10.1002/ejoc.201100077

27. Nakahata, M.; Takashima, Y.; Yamaguchi, H.; Harada, A. Nat. Commun. 2011, 2, No. 511. doi:10.1038/ncomms1521

28. Harada, A.; Kobayashi, R.; Takashima, Y.; Hashidzume, A.; Yamaguchi, H. Nat. Chem. 2011, 3, 34-37. doi:10.1038/nchem.893

29. Yamaguchi, H.; Kobayashi, R.; Takashima, Y.; Hashidzume, A.; Harada, A. Macromolecules 2011, 44, 2395-2399. doi:10.1021/ma200398y

30. Zheng, Y.; Hashidzume, A.; Takashima, Y.; Yamaguchi, H.; Harada, A. Langmuir 2011, 27, 13790-13795. doi:10.1021/la2034142

31. Yamaguchi, H.; Kobayashi, Y.; Kobayashi, R.; Takashima, Y.; Hashidzume, A.; Harada, A. Nat. Commun. 2012, 3, No. 603. doi:10.1038/ncomms 1617
32. Turro, N. J. Modern Molecular Photochemistry; University Science Books: Sausalito, CA, 1991.

33. Valeur, B. Molecular Fluorescence: Principles and Applications; Wiley-VCH: Weinheim, Germany, 2002.

34. Yorozu, T.; Hoshino, M.; Imamura, M. J. Phys. Chem. 1982, 86, 4426-4429. doi:10.1021/j100219a031

35. Hamai, S. J. Phys. Chem. 1989, 93, 6527-6529. doi:10.1021/j100354a048

36. Ueno, A. Supramol. Sci. 1996, 3, 31-36. doi:10.1016/0968-5677(96)00016-8

37. Zheng, Y.; Hashidzume, A.; Takashima, Y.; Yamaguchi, H.; Harada, A. Nat. Commun. 2012, 3, No. 831. doi:10.1038/ncomms1841

38. Siu, H.; Duhamel, J. J. Phys. Chem. B 2008, 112, 15301-15312. doi:10.1021/jp801105q

39. Siu, H.; Duhamel, J. J. Phys. Chem. B 2012, 116, 1226-1233. doi:10.1021/jp208168r

40. Duhamel, J. Polymers 2012, 4, 211-239. doi:10.3390/polym4010211 41. Duhamel, J. Langmuir 2012, 28, 6527-6538. doi:10.1021/la2047646

42. de Melo, J. S.; Costa, T.; da G. Miguel, M.; Lindman, B.; Schillén, K. J. Phys. Chem. B 2003, 107, 12605-12621. doi:10.1021/jp0346054

43. de Melo, J. S. S.; Costa, T.; Oliveira, N.; Schillén, K. Polym. Int. 2007, 56, 882-899. doi:10.1002/pi.2219

44. Blyshak, L. A.; Warner, I. M.; Patonay, G. Anal. Chim. Acta 1990, 232, 239-243. doi:10.1016/S0003-2670(00)81238-6

\section{License and Terms}

This is an Open Access article under the terms of the Creative Commons Attribution License (http://creativecommons.org/licenses/by/2.0), which permits unrestricted use, distribution, and reproduction in any medium, provided the original work is properly cited.

The license is subject to the Beilstein Journal of Organic Chemistry terms and conditions:

(http://www.beilstein-journals.org/bjoc)

The definitive version of this article is the electronic one which can be found at: $\underline{\text { doi: } 10.3762 / \text { bjoc } .8 .150}$ 